\title{
ABSOLUTELY ABNORMAL NUMBERS
}

\author{
GREG MARTIN
}

\section{INTRODUCTION}

A normal number is one whose decimal expansion (or expansion to some base other than 10) contains all possible finite configurations of digits with roughly their expected frequencies. More formally, when $b \geq 2$ is an integer, let

$$
N(\alpha ; b, a, x)=\#\{1 \leq n \leq x \text { : the } n \text {th digit in the base- } b \text { expansion of } \alpha \text { is } a\}
$$

denote the counting function of the occurrences of the digit $a(0 \leq a<b)$ in the $b$-ary expansion of the real number $\alpha$, and define the corresponding limiting frequency

$$
\delta(\alpha ; b, a)=\lim _{x \rightarrow \infty} x^{-1} N(\alpha ; b, a, x),
$$

if the limit exists. The number $\alpha$ is simply normal to the base $b$ if the limit defining $\delta(\alpha ; b, a)$ exists and equals $1 / b$ for each $0 \leq a<b$. (When $\alpha$ is a $b$-adic fraction $a / b^{n}$, which has one $b$-ary expansion with all but finitely many digits equaling zero and another $b$-ary expansion with all but finitely many digits equaling $b-1$, these limiting frequencies are not uniquely defined; however, such an $\alpha$ will not be simply normal to the base $b$ in either case.) A number is normal to the base $b$ if it is simply normal to each of the bases $b, b^{2}, b^{3}, \ldots$. This is equivalent (see [6, Chapter 8]) to demanding that for any finite string $b_{1} b_{2} \ldots b_{k}$ of base- $b$ digits, the limiting frequency of occurrences of this string in the $b$-ary expansion of $\alpha$ (defined analogously to equation (2) above) exists and equals $1 / b^{k}$.

For instance, it was shown by Champernowne [1] that the number $0.12345678910111213 \ldots$ formed by concatenating all of the positive integers together into a single decimal is normal to base 10 (the analogous construction works for any base $b \geq 2$ ), and this sort of example has been generalized [2, 3]. It is known that almost all real numbers are normal to any given base $b$ (see for instance [6, Theorem 8.11]), and consequently almost all real numbers are absolutely normal, i.e., normal to all bases $b \geq 2$ simultaneously. On the other hand, we have not proven a single naturally occurring real number to be absolutely normal.

Let us call a number abnormal to the base $b$ if it is not normal to the base $b$, and absolutely abnormal if it is abnormal to all bases $b \geq 2$ simultaneously. For instance, every rational number $r$ is absolutely abnormal: any $b$-ary expansion of $r$ will eventually repeat, say with period $k$, in which case $r$ is about as far from being simply normal to the base $b^{k}$ as it can be. Even though the set of absolutely abnormal numbers is the intersection of countably many sets of measure zero, it was pointed out by Maxfield [5] that the set of numbers normal to a given base $b$ is uncountable and dense; later, Schmidt [7] gave a complicated constructive proof of this fact. In this paper we exhibit a simple construction of a specific irrational (in fact, transcendental) real number that is absolutely abnormal:

Theorem. The number $\alpha$ defined in equation (8) below is irrational and absolutely abnormal.

1991 Mathematics Subject Classification. 11K16 (11A63). 
In fact, our construction easily generalizes to produce concretely an uncountable set of absolutely abnormal numbers in any open interval.

It is instructive to consider why constructing an irrational, absolutely abnormal number is even difficult. Since we already know that rational numbers are absolutely abnormal, our first thought might be to choose an irrational number whose $b$-ary expansions mimic those of rational numbers for long stretches, i.e., an irrational number with very good rational approximations. Thus a natural class to consider is the Liouville numbers, defined to be those real numbers $\beta$ such that for every positive integer $m$, there exists a rational number $\frac{p}{q}$ (not necessarily in lowest terms) satisfying

$$
0<\left|\beta-\frac{p}{q}\right|<\frac{1}{q^{m}}
$$

These Liouville numbers are all transcendcental (see Lemma 6 below) - in fact Liouville introduced these numbers precisely to exhibit specific transcendental numbers, and the offcited example

$$
\beta=\sum_{n=1}^{\infty} 10^{-n !}=0.11000100000000000000000100 \ldots
$$

is usually the first number that students see proven transcendental.

Clearly $\beta$ is abnormal to the base 10; how would we go about showing, for example, that $\beta$ is abnormal to the base 2 ? We would try to argue that the binary expansion of $\beta$ agrees with that of each of the rational numbers

$$
\beta_{k}=\sum_{n=1}^{k} 10^{-n !}
$$

through about the $\left((n+1)\right.$ ! $\left.\log _{2} 10\right)$-th binary digit. Since each $\beta_{k}$ is rational and thus abnormal to the base 2 , can we conclude that $\beta$ itself is abnormal to the base 2 ?

Not quite: it seems that we would have to show that there is a fixed power $2^{n}$ such that infinitely many of the $\beta_{k}$ were not simply normal to the base $2^{n}$. (For each $\beta_{k}$ there is some power $2^{n_{k}}$ such that $\beta_{k}$ is not simply normal to the base $2^{n_{k}}$, but these exponents $n_{k}$ might very well grow with $k$.) In fact, it is not hard to show (using the fact that 2 is a primitive root modulo every power of 5) that any 10-adic fraction that is not a 2-adic fraction-including each $\beta_{k}$-is simply normal to the base 2 ! In general, without actually computing binary expansions of specific fractions, it seems impossible to rule out the incredible possibility that the $\beta_{k}$ are accidentally simply normal to bases that are high powers of 2 . In summary, any Liouville number we write down is almost certain (morally) to be absolutely abnormal, but actually proving its absolute abnormality is another matter.

To circumvent this difficulty, we construct a Liouville number whose successive rational approximations are $b$-adic fractions with $b$ varying, rather than all being 10 -adic fractions as in equation (4). The existence of such Liouville numbers can certainly be proven using just the fact that the $b$-adic fractions are dense for any integer $b \geq 2$; however, our construction is completely explicit. We first give the complete construction of our irrational, absolutely abnormal number $\alpha$ and then show afterwards that $\alpha$ has the required properties. 


\section{The CONSTRUCTION AND PROOF}

We begin by defining a sequence of integers

$$
d_{2}=2^{2}, \quad d_{3}=3^{2}, \quad d_{4}=4^{3}, \quad d_{5}=5^{16}, \quad d_{6}=6^{30,517,578,125}, \ldots
$$

with the recursive rule

$$
d_{j}=j^{d_{j-1} /(j-1)} \quad(j \geq 3) .
$$

This sequence exhibits the pattern

$$
d_{4}=4^{3^{2-1}}, \quad d_{5}=5^{4^{\left(3^{2-1}-1\right)}}, \quad d_{6}=6^{5^{\left(4^{\left(3^{2-1}-1\right)}-1\right)}}, \ldots
$$

which in general gives the typesetting nightmare

$$
\left.d_{j}=j^{(j-1)}\left({ }_{(j-2)}\left({ }_{(j-3)}\left(.{ }^{\left(4^{\left(3^{2-1}\right)}-1\right)} \cdot .\right)_{-1}\right)\right)_{-1}\right) .
$$

Using these integers, we define the sequence of rational numbers

$$
\alpha_{k}=\prod_{j=2}^{k}\left(1-\frac{1}{d_{j}}\right)
$$

so that $\alpha_{2}=\frac{1}{4}, \alpha_{3}=\frac{2}{3}, \alpha_{4}=\frac{21}{32}, \alpha_{5}=\frac{100,135,803,222}{152,587,890,625}$, and so on.

We now nominate

$$
\alpha=\lim _{k \rightarrow \infty} \alpha_{k}=\prod_{j=2}^{\infty}\left(1-\frac{1}{d_{j}}\right)
$$

as our candidate for an irrational, absolutely abnormal number. The first few digits in the decimal expansion of $\alpha$ are

$$
\alpha=0.6562499999956991 \underbrace{99999 \ldots 99999}_{23,747,291,5599 \mathrm{~s}} 8528404201690728 \ldots,
$$

from which we can get an inkling of the extreme abnormality of $\alpha$ (at least to the base 10). We need to prove three things concerning this number $\alpha$ : first, that the infinite product (\$) defining $\alpha$ actually converges; second, that $\alpha$ is irrational; and finally, that $\alpha$ is absolutely abnormal.

It is apparent from the expressions (5) and (6) that the $d_{j}$ grow (ridiculously) rapidly and hence that the infinite product (8) should indeed converge. The following lemma provides a crude inequality relating the integers $d_{j}$ that we can use to prove this assertion rigorously.

Lemma 1. For $j \geq 5$ we have $d_{j}>2 d_{j-1}^{2}$.

Proof: We proceed by induction, the case $j=5$ being true by inspection. For $j>5$ we surely have

$$
d_{j}=j^{d_{j-1} /(j-1)}>5^{d_{j-1} /(j-1)} .
$$

Notice that from the definition (5) of the $d_{j}$,

$$
\frac{d_{j-1}}{j-1}=(j-1)^{\frac{d_{j-2}}{j-2}-1}>(j-1)^{\frac{d_{j-2}}{2(j-2)}}=\sqrt{d_{j-1}},
$$


and therefore

$$
d_{j}>5^{\sqrt{d_{j-1}}}
$$

Now using the fact (easily proven by your favorite calculus student) that $5^{x} \geq x^{5}$ for $x \geq 5$, we conclude that

$$
d_{j}>\left(\sqrt{d_{j-1}}\right)^{5}>2 d_{j-1}^{2}
$$

as desired.

Equipped with this inequality, we can now show that the infinite product (8) defining $\alpha$ converges. Moreover, we can show that the number $\alpha$ is well approximated by the rational numbers $\alpha_{k}$. (Notice that $\alpha_{4}$ is exactly 0.65625 and $\alpha_{5}$ is exactly 0.6562499999956992 - cf. the decimal expansion (9) of $\alpha$.)

Lemma 2. The product (8) defining a converges. Moreover, for $k \geq 2$ we have

$$
\alpha_{k}>\alpha>\alpha_{k}-\frac{2}{d_{k+1}}
$$

Proof: To show that the product (8) converges, we must show that the corresponding sum $\sum_{j=2}^{\infty} 1 / d_{j}$ converges. But by Lemma [ w we certainly have $d_{j}>2 d_{j-1}$ for $j \geq 5$, and therefore

$$
\sum_{j=2}^{\infty} \frac{1}{d_{j}} \leq \frac{1}{d_{2}}+\frac{1}{d_{3}}+\sum_{j=4}^{\infty} \frac{1}{2^{j-4} d_{4}}=\frac{1}{d_{2}}+\frac{1}{d_{3}}+\frac{2}{d_{4}}<\infty .
$$

Similarly, using the fact that $1 \geq \Pi\left(1-x_{j}\right) \geq 1-\sum x_{j}$ for any real numbers $0 \leq x_{j} \leq 1$, we see that for $k \geq 3$

$$
\begin{aligned}
\alpha_{k}>\alpha=\alpha_{k} \prod_{j=k+1}^{\infty}\left(1-\frac{1}{d_{j}}\right) & \geq \alpha_{k}\left(1-\sum_{j=k+1}^{\infty} \frac{1}{d_{j}}\right) \\
& >\alpha_{k}\left(1-\sum_{j=k+1}^{\infty} \frac{1}{2^{j-k-1} d_{k+1}}\right)=\alpha_{k}\left(1-\frac{2}{d_{k+1}}\right)>\alpha_{k}-\frac{2}{d_{k+1}} .
\end{aligned}
$$

The inequalities (10) for $k=2$ follow from those for $k=3$, as it is easily verified by hand that $\alpha_{2}>\alpha_{3}>\alpha_{3}-\frac{2}{d_{4}}>\alpha_{2}-\frac{2}{d_{3}}$.

It turns out that both the proof that $\alpha$ is irrational and the proof that $\alpha$ is absolutely abnormal hinge on the fact that each rational approximation $\alpha_{k}$ is in fact a $k$-adic fractionthat is, when $\alpha_{k}$ is expressed in lowest terms, its denominator divides a power of $k$. In other words, each time we multiply $\alpha_{k-1}$ by $1-\frac{1}{d_{k}}=\frac{d_{k}-1}{d_{k}}$ to obtain $\alpha_{k}$, the numerator of the latter fraction completely cancels out the denominator of $\alpha_{k-1}$, so that all that can remain in the denominator of $\alpha_{k}$ are the powers of $k$ present in $d_{k}$. Proving that this always happens is an exercise in elementary number theory, which we present in the next three lemmas.

Lemma 3. Let $k$ and $r$ be positive integers, and let $p$ be a prime. If $k$ is divisible by $p^{r}$, then $(k+1)^{p}-1$ is divisible by $p^{r+1}$. 
Proof: Writing $k=p^{r} n$, we have from the binomial theorem

$$
\begin{aligned}
(k+1)^{p}-1 & =\left(p^{r} n+1\right)^{p}-1 \\
& =\left\{\left(p^{r} n\right)^{p}+\left(\begin{array}{c}
p \\
p-1
\end{array}\right)\left(p^{r} n\right)^{p-1}+\cdots+\left(\begin{array}{c}
p \\
2
\end{array}\right)\left(p^{r} n\right)^{2}+\left(\begin{array}{c}
p \\
1
\end{array}\right) p^{r} n+1\right\}-1 \\
& =p^{r p} n^{p}+\left(\begin{array}{c}
p \\
p-1
\end{array}\right) p^{r(p-1)} n^{p-1}+\cdots+\left(\begin{array}{c}
p \\
2
\end{array}\right) p^{2 r} n^{2}+p \cdot p^{r} n .
\end{aligned}
$$

Since all of these binomial coefficients $\left(\begin{array}{l}p \\ k\end{array}\right)$ are integers, each term in this last sum is visibly divisible by $p^{r+1}$.

Lemma 4. For any positive integers $k$ and $m$, the integer $(k+1)^{k^{m}}-1$ is divisible by $k^{m+1}$.

Proof: If $p^{r}$ is any prime power dividing $k$, an $r m$-fold application of Lemma 3 shows us that $(k+1)^{p^{r m}}-1$ is divisible by $p^{r+r m}$. Then, since

$$
(k+1)^{k^{m}}-1=\left((k+1)^{p^{r m}}-1\right)\left((k+1)^{k^{m}-p^{r m}}+(k+1)^{k^{m}-2 p^{r m}}+\cdots+(k+1)^{p^{r m}}+1\right),
$$

we see that $(k+1)^{k^{m}}-1$ is also divisible by $p^{r(m+1)}$.

In particular, since $p^{r}$ was an arbitrary prime power dividing $k$, we see that $(k+1)^{k^{m}}-1$ is divisible by every prime power that divides $k^{m+1}$. This is enough to verify that $(k+1)^{k^{m}}-1$ is divisible by $k^{m+1}$ itself.

Lemma 5. For each $k \geq 2$, the product $d_{k} \alpha_{k}$ is an integer. In particular, since $d_{k}$ is a power of $k$, we see that $\alpha_{k}$ is a $k$-adic fraction.

Proof: We proceed by induction on $k$, the cases $k=2$ and $k=3$ being evident by inspection. For the inductive step, suppose (as our induction hypothesis) that $d_{k} \alpha_{k}$ is indeed an integer for a given $k \geq 3$. We may write

$$
d_{k+1} \alpha_{k+1}=\left(d_{k+1}-1\right) \alpha_{k}=\left((k+1)^{d_{k} / k}-1\right) \alpha_{k}=\frac{(k+1)^{d_{k} / k}-1}{d_{k}} \cdot d_{k} \alpha_{k}
$$

by the definitions (5) and (7) of $d_{k}$ and $\alpha_{k}$, respectively. The second factor $d_{k} \alpha_{k}$ is an integer by the induction hypothesis. On the other hand, we may rewrite

$$
(k+1)^{d_{k} / k}-1=(k+1)^{k^{d_{k-1} /(k-1)-1}}-1 .
$$

Applying Lemma ⿴囗十 with $m=d_{k-1} /(k-1)-1$, we see that this expression is divisible by $k^{d_{k-1} /(k-1)}=d_{k}$. Therefore the fraction on the right-hand side of equation (11) is in fact an integer, and so $d_{k+1} \alpha_{k+1}$ is itself an integer, which completes the proof.

As mentioned in the introduction, the key to proving that $\alpha$ is irrational is to show that it is in fact a Liouville number. It is a standard fact that any Liouville number is transcendental (see for instance [6, Theorem 7.9]); for the sake of keeping this paper self-contained, we include a proof.

Lemma 6. Every Liouville number is transcendental.

Proof: We shall prove the contrapositive, that no algebraic number can satisfy the Liouville property (3) for all positive $m$. Suppose that $\beta$ is algebraic. Without loss of generality, we may suppose that $|\beta| \leq \frac{1}{2}$ by adding an appropriate integer. Let

$$
m_{\beta}(x)=c_{d} x^{d}+c_{d-1} x^{d-1}+\cdots+c_{2} x^{2}+c_{1} x+c_{0}
$$


be the minimal polynomial for $\beta$, where the coefficients $c_{i}$ are integers. Now suppose that $\frac{p}{q}$ is a rational approximation to $\beta$, say $\left|\beta-\frac{p}{q}\right|<\frac{1}{2}$. Then

$$
\begin{aligned}
m_{\beta}\left(\frac{p}{q}\right) & =m_{\beta}\left(\frac{p}{q}\right)-m_{\beta}(\beta) \\
& =c_{d}\left(\left(\frac{p}{q}\right)^{d}-\beta^{d}\right)+\cdots+c_{2}\left(\left(\frac{p}{q}\right)^{2}-\beta^{2}\right)+c_{1}\left(\frac{p}{q}-\beta\right) \\
& =\left(\frac{p}{q}-\beta\right)\left(c_{d}\left(\left(\frac{p}{q}\right)^{d-1}+\left(\frac{p}{q}\right)^{d-2} \beta+\cdots+\beta^{d-1}\right)+\cdots+c_{2}\left(\frac{p}{q}+\beta\right)+c_{1}\right) .
\end{aligned}
$$

Since neither $\beta$ nor $\frac{p}{q}$ exceeds 1 in absolute value, we see that

$$
\left|m_{\beta}\left(\frac{p}{q}\right)\right| \leq\left|\frac{p}{q}-\beta\right| C(\beta),
$$

where we have defined the constant

$$
C(\beta)=d\left|c_{d}\right|+(d-1)\left|c_{d-1}\right|+\cdots+2\left|c_{2}\right|+\left|c_{1}\right|
$$

On the other hand, $m_{\beta}\left(\frac{p}{q}\right)$ is a rational number with denominator at most $q^{d}$, and it is nonzero since $m_{\beta}$ is irreducible. Therefore

$$
\left|m_{\beta}\left(\frac{p}{q}\right)\right| \geq \frac{1}{q^{d}}
$$

Together, the inequalities (12) and (13) imply that

$$
\left|\beta-\frac{p}{q}\right| \geq \frac{C(\beta)^{-1}}{q^{d}},
$$

which precludes the inequality (3) from holding when $m$ is large enough.

To show that $\alpha$ is indeed a Liouville number, we will need an inequality somewhat stronger than the one given in Lemma 1. The following lemma furnishes a simple inequality that is strong enough for this purpose.

Lemma 7. For $j \geq 5$ we have $d_{j+1}>d_{j}^{d_{j-1}}$.

Proof: It is immediate that

$$
d_{j+1}=(j+1)^{d_{j} / j}>j^{d_{j} / j}>j^{2 d_{j-1}^{2} / j}=\left(j^{d_{j-1} /(j-1)}\right)^{d_{j-1} \cdot 2(j-1) / j}=d_{j}^{d_{j-1} \cdot 2(j-1) / j}>d_{j}^{d_{j-1}},
$$

where we have used Lemma [1 for the second inequality.

Lemma 8. $\alpha$ is a Liouville number; in particular, $\alpha$ is transcendental.

Proof: We can easily show show that the $\alpha_{k}$ provide the very close rational approximations needed in equation (3) to make $\alpha$ a Liouville number. Indeed, $\alpha_{k}$ can be written as a fraction whose denominator is $d_{k}$ by Lemma 5, while Lemma 2 tells us that for $k \geq 5$

$$
0<\left|\alpha-\alpha_{k}\right|<\frac{2}{d_{k+1}}<\frac{2}{d_{k}^{d_{k-1}}},
$$

where the last inequality is by Lemma 7 . Since $d_{k-1}$ tends to infinity with $k$, this shows that $\alpha$ is a Liouville number (and hence transcendental by Lemma 6). 
At last we have all the tools we need to establish the theorem stated in the introduction:

Theorem. The number $\alpha$ defined in equation (8) is irrational and absolutely abnormal.

Proof: We have just shown in Lemma 8 that $\alpha$ is irrational. As for proving that $\alpha$ is absolutely abnormal, the idea is that for every integer base $b \geq 2$, the number $\alpha$ is just a tiny bit less than the $b$-adic fraction $\alpha_{b}$. Since the $b$-ary expansion of $\alpha_{b}$ terminates in an infinite string of zeros, the slightly smaller number $\alpha$ will have a long string of digits equal to $b-1$ before resuming a more random behavior. (This is evident in the decimal expansion (9) of $\alpha$, as $\alpha_{5}$ is a 10-adic fraction as well as a 5-adic fraction.) This happens more than once, as each of $\alpha_{b}, \alpha_{b^{2}}, \alpha_{b^{3}}$, and so on is a $b$-adic fraction. Consequently, the $b$-ary expansion of $\alpha$ will have increasingly long strings consisting solely of the digit $b-1$, which will prevent it from being even simply normal to the base $b$.

More quantitatively, let $b \geq 2$ and $r$ be positive integers. Since $d_{b^{r}} \alpha_{b^{r}}$ is an integer by Lemma 5, and since $d_{b^{r}}=\left(b^{r}\right)^{d_{b^{r}-1} /\left(b^{r}-1\right)}$ by definition, the $b$-ary expansion of $\alpha_{b^{r}}$ terminates after at most $r d_{b^{r}-1} /\left(b^{r}-1\right)$ nonzero digits. On the other hand, by Lemma 2 we know that $\alpha$ is less than $\alpha_{b^{r}}$ but by no more than $2 / d_{b^{r}+1}=2 /\left(b^{r}+1\right)^{d_{b^{r}} / b^{r}}<2 / b^{r d_{b^{r}} / b^{r}}$. Therefore, when we subtract this small difference from $\alpha_{b^{r}}$, the resulting $b$-ary expansion will have occurrences of the digit $b-1$ beginning at the $\left(r d_{b^{r}-1} /\left(b^{r}-1\right)+1\right)$-th digit at the latest, and continuing through at least the $\left(r d_{b^{r}} / b^{r}-1\right)$-th digit since the difference will start to show only in the

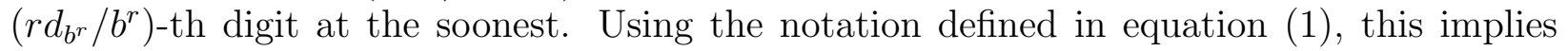
that

$$
N\left(\alpha ; b, b-1, \frac{r d_{b^{r}}}{b^{r}}\right) \geq \frac{r d_{b^{r}}}{b^{r}}-\frac{r d_{b^{r}-1}}{b^{r}-1}-1>\frac{r d_{b^{r}}}{b^{r}}-\frac{2 r d_{b^{r}-1}}{b^{r}} .
$$

At this point we can calculate that

$$
\begin{aligned}
\limsup _{x \rightarrow \infty} x^{-1} N(\alpha ; b, b-1, x) & \geq \limsup _{r \rightarrow \infty}\left(\frac{b^{r}}{r d_{b^{r}}} N\left(\alpha ; b, b-1, \frac{r d_{b^{r}}}{b^{r}}\right)\right) \\
& \geq \limsup _{r \rightarrow \infty}\left(1-\frac{2 d_{b^{r}-1}}{d_{b^{r}}}\right) .
\end{aligned}
$$

Using Lemma 1, we see that

$$
\limsup _{x \rightarrow \infty} x^{-1} N(\alpha ; b, b-1, x) \geq \limsup _{r \rightarrow \infty}\left(1-\frac{2 d_{b^{r}-1}}{2 d_{b^{r}-1}^{2}}\right)=\limsup _{r \rightarrow \infty}\left(1-\frac{1}{d_{b^{r}-1}}\right)=1 .
$$

In particular, the frequency $\delta(\alpha ; b, b-1)$ defined in equation (2) either does not exist or else equals 1 , either of which precludes $\alpha$ from being simply normal to the base $b$. Since $b \geq 2$ was arbitrary, this shows that $\alpha$ is absolutely abnormal.

\section{Generalizations AND FURTher QUeStions}

We mentioned in the introduction that our construction of an irrational, absolutely abnormal number can be generalized to exhibit an uncountable set of absolutely abnormal numbers in any open interval, and we now describe that extension. Clearly we may limit our attention to subintervals of $[0,1]$, since the set of normal numbers to any base is invariant under translation by an integer. In the original construction at the beginning of Section 2, we began with $d_{2}=2^{2}$ and $\alpha_{2}=\frac{3}{4}$; to be more general, let $\alpha_{2}$ be any 2 -adic fraction $\frac{a}{d_{2}}$, where $d_{2}=2^{n_{2}}$ for some positive integer $n_{2}$. Next fix any sequence $n_{3}, n_{4}, \ldots$ of positive 
integers and modify the recursive definition (5) of the $d_{j}$ to

$$
d_{j}=j^{n_{j} d_{j-1} /(j-1)} \quad(j \geq 3) .
$$

If we now set

$$
\alpha_{k}=\alpha_{2} \prod_{j=3}^{k}\left(1-\frac{1}{d_{j}}\right)
$$

(where of course the numbers $\alpha_{k}$ now depend on $\alpha_{2}$ and the $n_{j}$ ), then the presence of the integers $n_{j}$ will not hinder the proof of Lemma 5 that $d_{k} \alpha_{k}$ is always a $k$-adic fraction (this is a consequence of the fact that $x-1$ always divides $x^{n}-1$ ). Therefore the new limit $\alpha=\lim _{k \rightarrow \infty} \alpha_{k}$ can be shown to be a transcendental, absolutely abnormal number in exactly the same way, the modifications only accelerating the convergence of the infinite product and enhancing the ease with which the various inequalities in the Lemmas are satisfied. (When we make this modification, then the one case we must avoid is $\alpha_{2}=\frac{1}{2}$ and $n_{2}=n_{3}=\cdots=1$, for in this case it will happen that $d_{j}=j^{1}$ for every $j \geq 2$. Then each product $\alpha_{k}$ is a telescoping product with value $\frac{1}{k}$, and their limit $\alpha=0$, while certainly absolutely abnormal, will be uninterestingly so.)

In particular, Lemma 2 applied with $k=2$ would show in this context that $\frac{a}{2^{n_{2}}}>\alpha>\frac{a-2}{2^{n_{2}}}$; thus by choosing $\alpha_{2}=\frac{a}{2^{n_{2}}}$ appropriately, we can ensure that the resulting number $\alpha$ lies in any prescribed open subinterval of $[0,1]$. Moreover, the various choices of the integers $n_{3}$, $n_{4}, \ldots$ will give rise to distinct limits $\alpha$; one can show this by considering the first index $j \geq 3$ at which the choices of $n_{j}$ differ, say, and then applying Lemma 2 with $k=j$ to each resulting $\alpha$. This generalization thus permits us to construct uncountably many transcendental, absolutely abnormal numbers in any prescribed open interval.

One interesting special case of this generalized construction arises from the choices $\alpha_{2}=\frac{1}{2}$ and $n_{j}=\phi(j-1)$ for all $j \geq 3$, where $\phi$ is the Euler totient function; these choices give the simple recursive rule $d_{j}=j^{\phi\left(d_{j-1}\right)}$ for $j \geq 3$. In this special case, the crucial property that $d_{k} \alpha_{k}$ is always an integer is in fact a direct consequence of Euler's theorem that $a^{\phi(n)}$ is always congruent to 1 modulo $n$ as long as $a$ and $n$ have no common factors. In general, the smallest exponent $e_{k}$ we can take in the recursive rule $d_{k}=k^{e_{k}}$ so that this crucial property is satisfied is the multiplicative order of $k$ modulo $n$, which might be smaller than $d_{k-1} /(k-1)$; however, our construction given in Section 2 has the advantage of being more explicit, as it is not necessary to wait and see the exact value of $d_{k-1}$ before knowing how we will construct $d_{k}$.

We remark that Schmidt's construction [7] mentioned in the introduction actually gives the following more powerful result: given any set $S$ of integers exceeding 1 with the property that an integer $b$ is in $S$ if and only if every perfect power of $b$ is in $S$, Schmidt constructed real numbers that are normal to every base $b \in S$ and abnormal to every base $b \notin S$. (The problem considered herein is the special case where $S=\emptyset$.) It would be interesting to see if the construction in this paper could be modified to produce these "selectively normal numbers" as well.

We conclude with a few remarks about absolutely simply abnormal numbers, numbers that are simply normal to no base whatsoever. As we saw in the proof of our Theorem, the number $\alpha$ does in fact meet this stronger criterion of abnormality. On the other hand, while all rational numbers are absolutely abnormal, many of them are in fact simply normal to various bases. For example, $1 / 3$ is simply normal to the base 2 , as its binary representation is 
$0.010101 \ldots$ In fact, one can check that every fraction in reduced form whose denominator is $3,5,6,9,10,11,12,13,17,18,19,20, \ldots$ is simply normal to the base 2 (presumably there are infinitely many such odd denominators - can it be proven?); every fraction whose denominator is $7,14,19,21,31, \ldots$ is simply normal to the base 3 ; and so on. Somewhat generally, if $p$ is a prime such that one of the divisors $b$ of $p-1$ is a primitive root for $p$, then every fraction whose denominator is $p$ is simply normal to the base $b$ (although this is not a necessary condition, as the normality of fractions with denominator 17 to base 2 shows).

For a fraction with denominator $q$ to be simply normal to the base $b$ (we can assume, by multiplying by $b$ a few times if necessary, that $b$ and $q$ are relatively prime), it is necessary for $b$ to divide the multiplicative order of $b$ modulo $q$, and hence $b$ must certainly divide $\phi(q)$ by Euler's theorem. Therefore, we immediately see that every fraction whose denominator is a power of 2 is absolutely simply abnormal. One can also verify by this criterion that every fraction whose denominator in reduced form is 15 or 28 , for example, is absolutely simply abnormal. It seems to be a nontrivial problem to classify, in general, which rational numbers are absolutely simply abnormal. Since some fractions with denominator 63 are simply normal to the base 2 while others are not, as one can check, absolute simple abnormality probably depends in general on the numerator as well as the denominator of the fraction.

Acknowledgements: Glyn Harman gave a survey talk on normal numbers at the Millennial Conference on Number Theory at the University of Illinois in May 2000 (see [1]), at the end of which Andrew Granville asked the question about a specific absolutely abnormal number that spurred this paper. Carl Pomerance suggested the number $\sum_{n=1}^{\infty}(n !)^{-n !}$, a Liouville number which again is almost surely absolutely abnormal, but a proof of this seems hopeless. The author would like to thank all three for their interest in this construction, and also an anonymous referee for several helpful comments on a preliminary version of this paper.

\section{REFERENCES}

[1] D.G. Champernowne, The construction of decimals normal in the scale of ten, J. London Math. Soc. 8 (1933), 254-260.

[2] A.H. Copeland and P. Erdős, Note on normal numbers, Bull. Amer. Math. Soc. 52 (1946), 857-860.

[3] H. Davenport and P. Erdős, Note on normal decimals, Canadian J. Math. 4 (1952), 58-63.

[4] G. Harman, 100 years of normal numbers, Proceedings of the Millennial Conference on Number Theory (Urbana, IL), A K Peters, (to appear).

[5] J.E. Maxfield, Normal k-tuples, Pacific J. Math. 3 (1953), 189-196.

[6] I. Niven, Irrational numbers, The Mathematical Association of America. Distributed by John Wiley and Sons, Inc., New York, N.Y., 1956, The Carus Mathematical Monographs, No. 11.

[7] W.M. Schmidt, Über die Normalität von Zahlen zu verschiedenen Basen, Acta Arith. 7 (1961/1962), 299-309.

Department of Mathematics, University of Toronto, Canada M5S 3G3

E-mail address: gerg@math.toronto.edu 